TIFR/TH/01-31

\title{
Percolation Systems away from the Critical Point
}

\author{
Deepak Dhar \\ Tata Institute of Fundamental Research, \\ Homi Bhabha Road, Mumbai 400 005, INDIA \\ e-mail: ddhar@theory.tifr.res.in
}

\begin{abstract}
$\underline{\text { ABSTRACT }}$
This article reviews some effects of disorder in percolation systems even away from the critical density $p_{c}$. For densities below $p_{c}$, the statistics of large clusters defines the animals problem. Its relation to the directed animals problem and the Lee-Yang edge singularity problem is described. Rare compact clusters give rise to Griffiths singuraties in the free energy of diluted ferromagnets, and lead to a very slow relaxation of magnetization. In biassed diffusion on percolation clusters, trapping in dead-end branches leads to asymptotic drift velocity becoming zero for strong bias, and very slow relaxation of velocity near the critical bias field.
\end{abstract}

\section{Introduction}

Let me start by thanking Professor H. R. Krishnamurthy and other members of the organizing committee for inviting me to this meeting to felicitate Professor Narendra Kumar on his 60th birthday, and giving me an opportunity to pay my tribute to him.

Over years, I have always enjoyed discussing various questions with Narendra. The large spectrum of his interests, and his spirit of enquiry, and insights to disentangle the essential problem from confusing camouflage have been a source of admiration for me. Thus I am really very happy to come here, and express my respect for him, and join the other speakers in wishing him many more years of happy questing.

While I have shared with Kumar a common interest in understanding disordered systems, my own work has been largely in classical statistical mechanics $(\hbar=0)$, while Kumar's contributions to quantum problems have been discussed by several speakers here. Even so, I can legitimately claim to be one of Kumar's coworkers. Our only paper together was on the behavior of $\pm J$ spin-glass on a Bethe lattice, and was presented at the DAE Solid State Physics Symposium meeting at Madras ( now Chennai) in December, 1979. It turned out that neither Narendra nor I could attend the meeting, so the paper was presented by our host, 
Profesor Krishnamurthy. The abstract of the paper appeared in the proceedings of the symposium. Unfortunately, both of us got enmeshed in other problems, and did not ever write up this work for publication in regular journals.

Professor Krishnamurthy has suggested that it would be better if I discuss generally the different effects of disorder in classical statistical mechanics, and not narrowly focus on the topic of my latest preprint. I was rather relieved by this, as my paper-writing has been even slower than my usual (slow) rate in the recent past. So, in this talk, I will not describe any new results, but discuss some examples of interesting effects caused by disorder in a classical percolation system. The selection of topics was determined primarily by my familiarity rather than any other reasons. I will only outline the main results. The interested reader will have to go back to the cited literature for details.

Consider a random mixture of equal-sized conducting and insulating beads in a box. It is easy to see that as the fraction of conducting balls is varied from zero to one, the bulk mixture undergoes a transition from insulating to conducting. This is the basic percolation transition, and has been studied a lot as a simple geometrical model of phase transitions. As in the case of thermal critical phase transitions, various physical quantities have singular behavior near the phase transition, characterized by "critical exponents". Scaling theory, simulations and theoretical techniques such as renormalization group etc. have been used very successfully to understand the behavior of percolation systems at its critical point. In particular, in two dimensions, all the critical exponents of percolation are known from conformal field theory. Several good reviews of this subject are now available [1].

There is more to percolation theory than the critical exponents. Of course, an experimental disordered system may often be modelled by site- or bond- percolation. In general, one is much more likely to find it not near its percolation threshold. Critical exponents of the percolation theory are not of much use in describing these systems. It turns out that percolation systems, as classical models of disordered media show many interesting features, even away from the critical point. I will discuss some examples of these in the following.

\section{Off-critical Exponents in Percolation}

Let $P_{n}(p)$ be be probability that the cluster of connected sites containing the origin has exactly $n$ sites. Then, for all $p<p_{c}$, for large $n$,

$$
P_{n}(p) \sim A n^{-\theta} \exp [-B(p) n]
$$

where $\mathrm{B}(\mathrm{p})$ is a $\mathrm{p}$-dependent function that goes to zero as $p$ tends to $p_{c}$. The exponent $\theta$ is independent of $p$, and depends only on the dimension of space. This law is valid for $n$ much greater than the typical cluster size $n^{\star}(p)$. For $n \ll n^{\star}(p)$, one gets a different exponent $P_{n}(p) \sim n^{-\tau}$, with $\tau \neq \theta$. As $p$ tends to $p_{c}, n^{\star}(p)$ 
diverges. Here $\tau$ is a critical exponent of percolation theory, but $\theta$ is an off-critical exponent.

In the limit $p \rightarrow 0$, all clusters of $n$ sites have the equal weight $\left(p^{n}\right)$. Let $A_{n}$ be the number of different clusters of $n$ sites possible that contain the origin. (These are called rooted animals: same cluster with different positions of origin are counted as distinct [2].) Then one expects that for large $n$,

$$
A_{n} \simeq K \lambda^{n} n^{-\theta}
$$

The exponent $\theta$ in this equation is same as that defined by Eq.(四), because for small $p, P_{n}(p) \sim A_{n} p^{n}$. One can also define the average linear size of an animal of $N$ sites. This grows as $n^{\nu}$, where the exponent $\nu$ is related to the exponent $\theta$ defined above by the relation

$$
\theta=(d-2) \nu
$$

where $d$ is the dimension of space. The above equation is valid for $1 \leq d \leq$ 8. For $d \geq 8$, the exponents $\theta$ and $\nu$ stick to their mean-field values $3 / 2$ and $1 / 4$ respectively. Eq.(3) has the form of a hyperscaling relation except that $(d-2)$ appears here instead of $d$. This is understood as being due to a hidden supersymmetry in the problem [3], which makes the problem of determining the number of animals in $d$-dimensions related to the problem of Lee-Yang edge singularity in $(d-2)$ dimension.

The Lee-Yang description of the mathematical mechanism of phase transitions is well-known [4]. For a hard-core lattice gas in a finite volume, with possible additional attractive short-ranged interactions, the grand-canonical partition function is a finite degree polynomial with positive coefficients in the chemical activity $z$. The zeroes of this polynomial often (not always) lie on lines in the complex- $z$ plane. As the temperature is varied, the coefficients of the polynomial change, and the zeroes move. If at some temperature, the zeroes come arbitrarily close to the real axis as the size of the system is increased, the free energy per site becomes a non-analytic function of $z$, signalling the onset of a phase-transition.

The density of zeroes along such a line of zeroes near its end point shows a power-law dependence on the distance from the endpoint. We define the LeeYang edge singularity exponent $\sigma$ by the relation that the density varies as $\epsilon^{\sigma}$ at distance $\epsilon$ from the endpoint. It turns out that $\sigma$ is independent of temperature for all temperatures $T$ above the criticial temperature $T_{c}$, and depends only on the dimension of the system. It was shown by Parisi and Sourlas [3] that the animal exponent $\theta(d)$ in $d$-dimensions is related to the Lee-Yang edge singularity exponent in $d-2$ dimensions

$$
\theta(d)=\sigma(d-2)+1
$$

If we allow only neighbors in the "forward direction" , ( say along the direction of increasing coordinates on a hypercubical lattice), we get animals with a 
directional constraint. It turns out [5] that critical exponents of directed animals in $d$ dimensions turn out to be related to those of undirected animals in $(d+1)$ dimensions.

$$
\theta_{\text {dir }}(d)=\theta(d+1)
$$

and the transverse size exponent $\nu_{\perp, d i r}$ for directed animals in $d$ dimensions is the same as the (only one) size exponent $\nu$ for undirected animals in $(d+1)$ dimensions

$$
\nu_{\perp, d i r}(d)=\nu(d+1) .
$$

The exponent $\sigma$ is easily shown to take the values $\sigma=-1$ for $d=0$ ( a point), and $\sigma=-1 / 2$ for $d=1$. For $d=2$, one can use the exact solution of hard hexagon lattice gas by Baxter to show that $\sigma=-1 / 6$ for $d=2$ [6]. This then shows that the exact values of the exponent $\theta$ for undirected animal in dimensions $1,2,3,4$ are $-1,0, \frac{1}{2}, \frac{5}{6}$ respectively. The corresponding values of the size exponent $\nu$ are $1, \frac{1}{2}, \frac{5}{12}$ in dimensions $1,3,4$ respectively. In two dimensions, the exponent $\nu$ is not determined by scaling relations given above. The upper critical dimension for the animals problem is 8 , and for all $d \geq 8$, we get $\theta=\frac{3}{2}$, and $\nu=\frac{1}{4}$. The exponents for directed animals are easily determined from the scaling relations given above.

We have seen that there is a fairly good understanding of the off-critical "below $p_{c}$ " exponents of percolation. One can also define off-critical exponents in the super-critical regime of percolation theory. It was shown by Kunz and Suillard [7] that for all $p>p_{c}$, the probability that the origin belongs to a finite cluster of $n$ sites varies as $\exp \left(-b(p) n^{\frac{d-1}{d}}\right)$, for sufficiently large $s$. This is easy to understand: to get a finite cluster of $n$ sites, we need to disconnect a it from the infinite cluster. This needs perimeter bonds order $\left(n^{\frac{d-1}{d}}\right)$ bonds, which gives the result. More accurately, there is apower-law prefactor multiplying the exponential term, and the probability for a finite cluster of $n$ sites varies as

$$
P_{n}(p) \sim K n^{-\theta^{\prime}} \exp \left(-b(p) n^{\frac{d-1}{d}}\right)
$$

where $b(p)$ is a function of $p$, and $K$ is a constant. The exponent $\theta^{\prime}$ can be calculated exactly [8] using the fact that such clusters are roughly compact, with linear size varying as $n^{\frac{1}{d}}$, and the fluctuations in the $(d-1)$-dimensional roughly spherical surface can be described in terms of normal modes of vibration of the surface. Lubensky and McKane using field theory techniques showed that the exponent $\theta^{\prime}$ takes the values $\frac{5}{4},-\frac{1}{9}, \frac{1}{8},-\frac{449}{450},-\frac{11}{12}$ for $d=2,3,4,5,6$ respectively. The non-monotonic behavior of $\theta^{\prime}$ as a function of $d$ comes from the fact that the integrals over normal modes in odd and even dimensions coming in the theory have different behaviors.

Note that this is one of the few cases where non-trivial values of exponents can be exactly calculated in many dimensions greater than 2 . 


\section{Relaxation in Disordered Ferromagnets}

Consider now the case when the atom at each occupied site in a percolation network carries a magnetic moment, and there is a nearest neighbor ferromagetic interaction $J$ between the magnetic atoms. The "unoccupied" sites may be vacancies, or occupied by nonmagnetic atoms. Let $p$ denote the concentration of magnetic atoms. Then if $p<p_{c}$, the percolation threshold, then the system breaks up into mutually-disconnected clusters of magnetically coupled spins. In such a system no long-range spontaneous magnetization is possible at any nonzero temperature. For $p>p_{c}$, there is an infinite connected cluster, and at sufficiently low temperatures, spontaneous magnetization exists. The transition temperature $T_{\text {curie }}(p)$ depends on $p$, and goes to zero, as $p$ is decreased from 1 to $p_{c}$. The ferromagnetic phase is denoted by $\mathrm{F}$ in Fig. 1.

For $p<p_{c}$, there is no spontaneous magnetization. Let $f(T, h)$ be the disorder-averaged free energy per site of this system at a temperature $T$ in a magnetic field $h$. However, it was shown by Griffiths [9] that $f(T, h)$ is a nonanalytic function of the magnetic field at $h=0$ for all $T<T_{\text {curie }}(p=1)$. While the partial derivatives $\frac{\partial^{n}}{\partial h^{n}} f(T, h)$ exist for all positive integers $n$, and are finite, the Taylor series for $f(T, h)$ in powers of $h$ does not converge for any $T$ below the $T_{\text {curie }}(p=1)$, the Curie-temperature of the "pure" system. Thus, in the entire region marked $\mathrm{G}$ ( for the Griffiths phase) in fig. 1, there is no spontaneous magnetization, but the free energy per site $f(T, h)$ is a non-analytic function of $h$.

While the nonanalyticity in $f(T, h)$ as a function of $h$ is of the nature of an essential singularity, and is difficult to verify in experimental systems, the rare large clusters responsible for it have a much more pronounced effect on the rate of relaxation to equilibrium in disordered systems. Consider the decay of magnetization in such a system. We assume that the system is coupled to heat bath at temperatute $T<T_{\text {curie }}(p=1)$, and relaxes by single-spin -flip Glauber dynamics. At time $t=0$, the system is prepared in a state with all spins up. We monitor the bulk magnetization $M(t)$ at large times $t$. Since the spins in different clusters do not interact with each other, we can write $M(t)$ as a weighted sum over different cluster configurations $\mathcal{C}$.

$$
M(t)=\sum_{C} \operatorname{Prob}(\mathcal{C})<S(t)>_{\mathcal{C}}
$$

For a given finite cluster $\mathcal{C}$ of $n$-sites, one can determine the average magnetization at a site in the cluster by explicit diagonalization of the of matrix of size $2^{n} \times 2^{n}$. This is non-trivial, except for very small $n$. Fortunately, the behavior of $M(t)$ for large $t$ can be determined by simple qualitative arguments.

In the summation Eq.(8), the leading behavior of each term is exponential in time, with the decay rate $\gamma_{\mathcal{C}}$ depends on the cluster $\mathcal{C}$. Thus, we may write

$$
<S(t)>_{\mathcal{C}} \simeq \exp \left(-\gamma_{\mathcal{C}} t\right)
$$




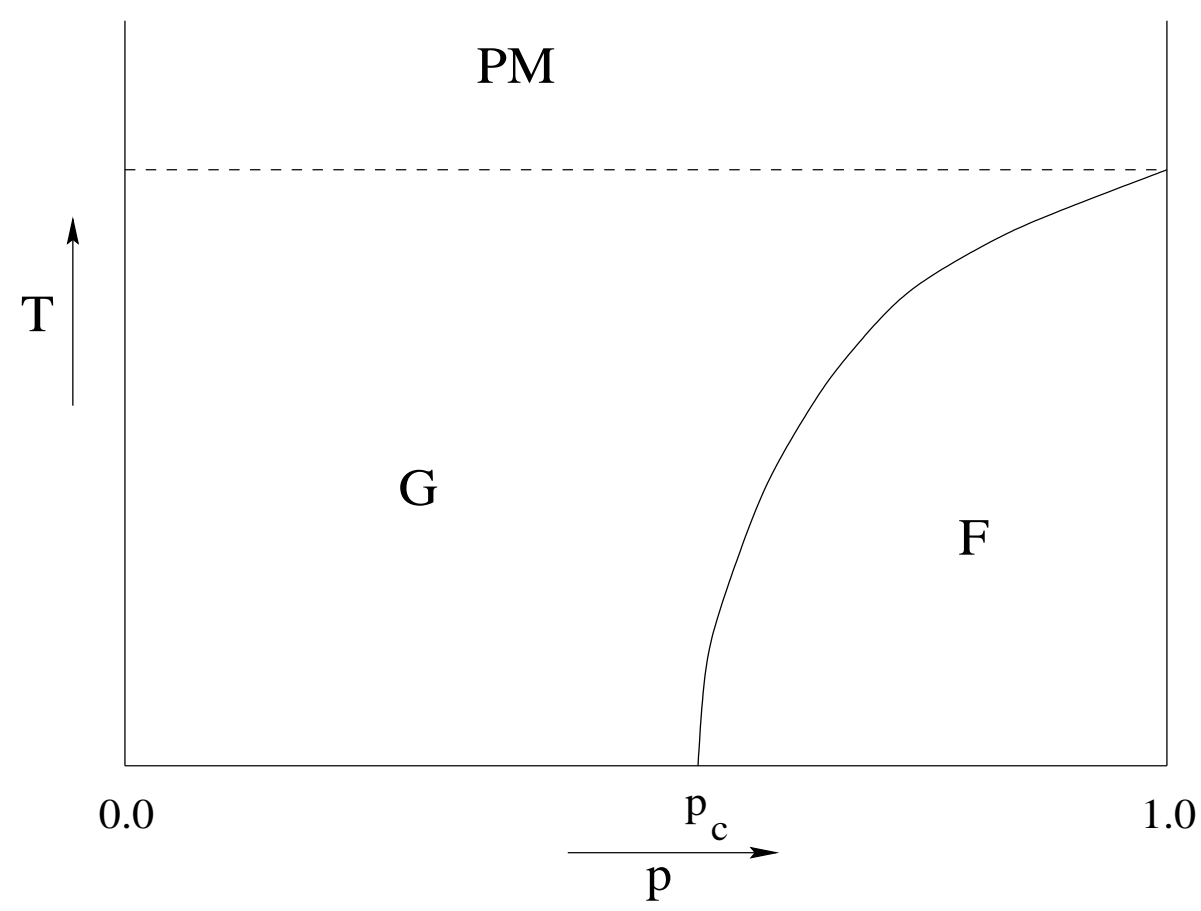

Figure 1:

and hence

$$
M(t) \simeq \sum_{C} \operatorname{Prob}(\mathcal{C}) \exp \left(-\gamma_{\mathcal{C}} t\right)
$$

At large times, clusters with smallest decay rates contribute most. The slowest relaxing clusters are those where all sites within a disc of radius $R$ are occupied. The density of such clusters varies as $\exp \left(-A R^{d}\right)$, where the constant $A$ depends on $p$. If the magnetization of such a cluster has to flip, it would need to create a domain wall of energy $\simeq \sigma R^{d-1}$. The rate of such activated process will decrease for large $R$ as $\exp \left(-\sigma R^{d-1} / T\right)$. It is then straight forward to put these estimates in the Eq.(8), and deduce that

$$
M(t) \sim \exp \left(-K(\log t)^{\frac{d}{d-1}}\right), \quad \text { for large } \mathrm{t} .
$$

For $p>p_{c}$, in the ferromagnetic phase (F in fig. 1) the probability of a large finite cluster of radius $R$, varies as $\exp \left(-a R^{d-1}\right)$, and not as $\exp \left(-R^{d}\right)$. Then the steepest descent calculation shows that in the ferromagnetic phase $F$, the magnetization at long times decays as a power law $M(t) \sim t^{-c}$, where the exponent $c$ depends on both $p$ and $T$.

The argument outlined above was first presented in [10]. The argument has been refined [11, 12]. Unfortunately, neither actual experimental data on disordered ferromagnets, nor results of numerical simulations [13] show a clear evidence of such a $\exp \left[-(\log t)^{x}\right]$ behavior. Presumably the time scale beyond which the 
contribution of rare clusters will dominate is larger than experimentally accessible time-scales. The experimental data seems to fit better a stretched exponential $\exp \left(-t^{x}\right)$. It seems that a more careful argument, that gives not only the correct asymptotic behavior at longest times, but also at intermediate times is needed.

\section{Biassed Diffusion on Percolation Networks}

Consider the motion of a single diffusing particle on a percolation network, say in two dimensions, with density of occupied sites being $p$. We assume that the particle can move only on the occupied sites of the lattice. Then if $p$ is less than the critical probability $p_{c}$, the particle is localized. For $p>p_{c}$, if the particle starts on the infinite cluster, its mean square deviation from the initial position grows linearly with time, $\left\langle R^{2}>\sim D(p) t\right.$, where the diffusion constant $D(p)$ depends on $p$ and tends to zero as $p$ tends to $p_{c}$. This problem of (unbiassed) diffusion on percolation clusters has been studied a lot [14].

If there is a larger probability of displacement in some direction, due to an imposed field, we have biassed diffusion. We shall model it by assuming that at any time the diffusing particle attempts to take a step in the up, right, down, left directions with probabilities $(1-B) / 4,1 / 4,(1+B) / 4$ and $1 / 4$ respectively. The step is actually taken if the intended destination site is occupied. If the biassing field $B$ is small, we have a non-zero value of average displacement per step, and this gives rise to mean displacement in time $t$ in the direction of the field growing linearly with $t$, and the mean velocity in time time $t$ tends to a constant

$$
\vec{v}_{\infty}=\operatorname{Lim}_{t \rightarrow \infty}<\vec{R}_{t}>/ t
$$

This asymptotic velocity $\vec{v}_{\infty}$ is proportional to $B$ for small $B$.

If $p$ is near 1 , most of the sites are occupied, and at large length scales, the medium looks homogenous. One then expects that so long as $p>p_{c}$, we expect the same behavior as in the system without disorder $(p=1)$.

Bottger and Bryksin realized that this is not so [15]. They argued that the mean velocity must tend to zero as $B$ tends to 1 , because of the possibility of trapping in dead-ends. We argued in [16], that for any $p<1$, there exists a critical bias $B_{c}$ such that the asymptotic drift velocity $\vec{v}$ is exactly zero for all $B>B_{c}$. This is easily seen: during its motion, the particle may get trapped in dead-end branches for long times, as it has to move against the field to get out of the trap. For a trap of depth $\ell$ the potential barrier to cross increases with $\ell$, and the trapping time varies as $\left(\frac{1+B}{1-B}\right)^{\ell}$. The density $\rho(\ell)$ of traps of depth $\ell$ varies as $\exp (-\ell / \xi)$. Hence the average trapping time per step along the backbone is

$$
\sum_{\ell=1}^{\infty} \rho(\ell)\left(\frac{1+B}{1-B}\right)^{\ell}
$$




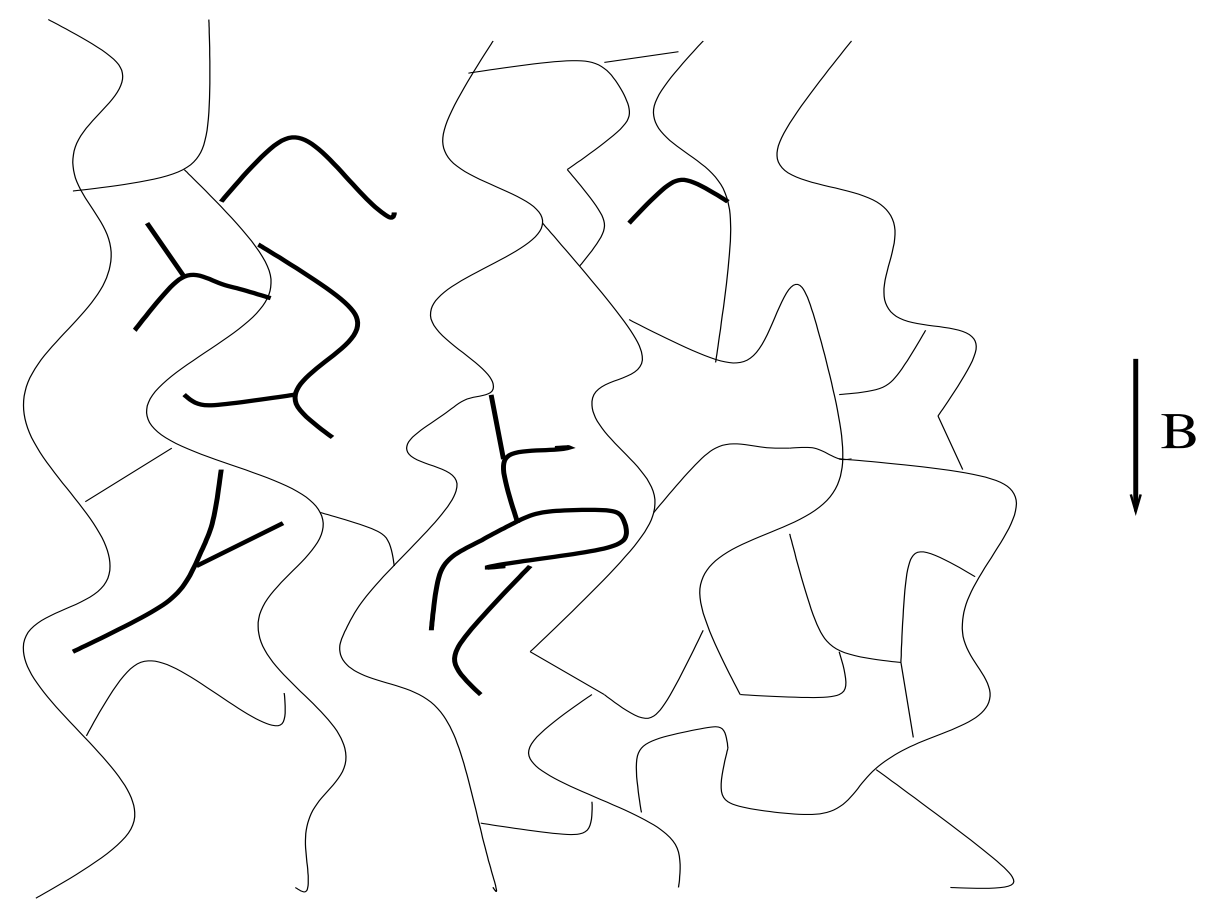

Figure 2:

where $\xi$ is the $p$-dependent percolation correlation length of the system. This summation converges only for $B<B_{c}=\tanh \left(\frac{1}{2 \xi}\right)$. For $B=B_{c}-\epsilon$, with $\epsilon>0$, this summation varies as $1 / \epsilon$, and the mean velocity, which varies inversely as the the mean trapping time varies proportional to $\epsilon$.

For $B>B_{c}$, the asymptotic velocity $\vec{V}_{\infty}$ is zero, but the mean displacement of the particle $\left\langle\vec{R}_{t}>\right.$ increases as $t^{a}$ with $a<1$. The exponent $a$ depends continuously on $B$, and is easily obtained by using the condition that the density of traps with trapping time geater than or equal to $t$ varies as $t^{-a}$, where

$$
a=\left(\frac{1}{\xi}\right) \log \left(\frac{1+B}{1-B}\right)
$$

In a time $t$, the particle can, on the average, only travel a distance $t^{a}$ before it encounters a trap with trapping time bigger than $t$, and gets stuck there. Eventually, it will exit from this trap, only to get stuck in other traps, some with an even larger trapping times. Thus, if we examine all particles at some large time $t$, typically they would be stuck in, or just emerging from a trap with trapping time of order $t$.

For $B=B_{c}$, the average trapping time per step encountered by the walker is given by (13), except that summation over $\ell$ is cutoff at a value $\ell_{\max }$, where $\ell_{\max }$ is the typical value of the trapping time of the deepest trap encountered by a walker upto time $t$. It is easy to see that $\ell_{\max }$ varies as logt for large $t$, and hence the average velocity of the particle up to time $t$ varies as $\frac{1}{\text { logt }}$. Thus the average 


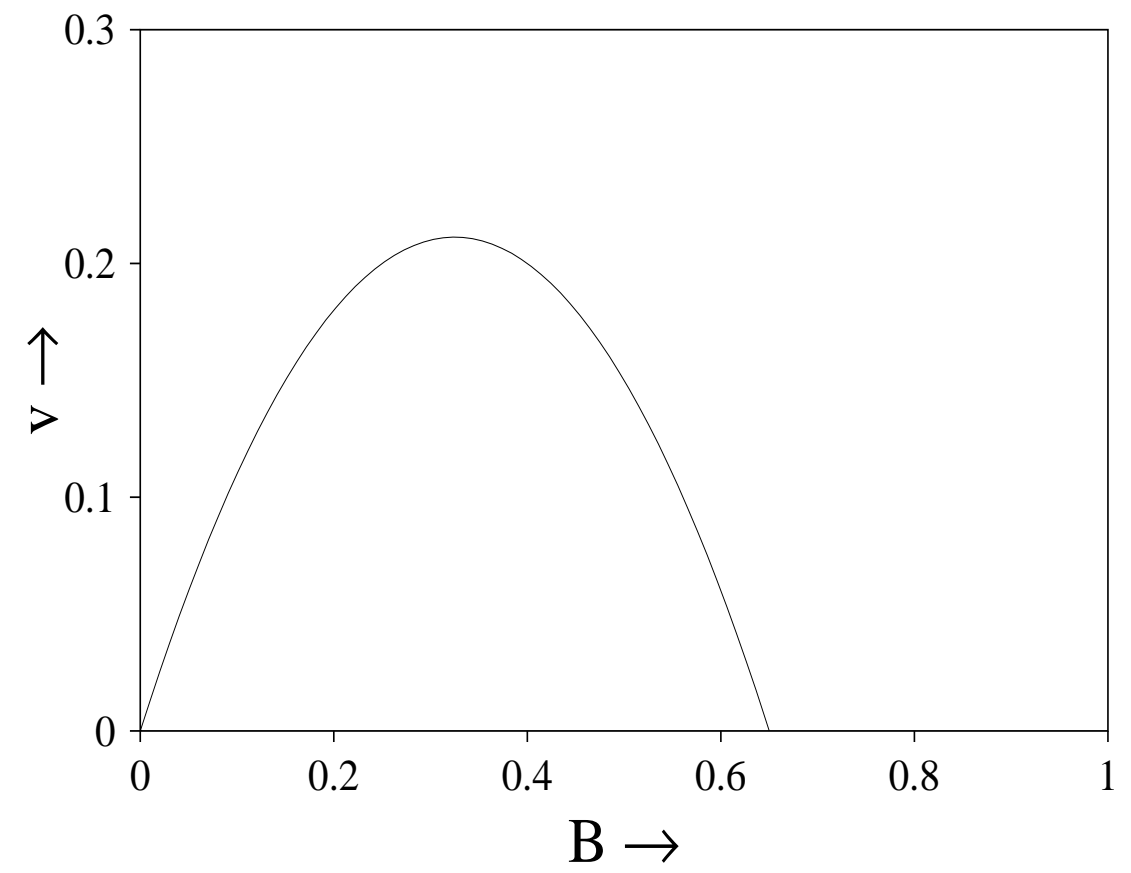

Figure 3:

velocity decreases very slowly to zero at $B=B_{c}$. This very slow relaxation has been checked in large-scale simulations of this problem [17].

If $B=B_{c}-\epsilon$, then this slow decay of the velocity continues from its initial value of order 1 , to the final value which is of order $\epsilon$. As the initial decay of velocity would be nearly same as that for $B=B_{c}$, we see that typical relaxation time $\tau(B)$ for the average velocity in an ensemble of non-interacting particles to reach the steady state value varies as

$$
\tau(B) \sim \exp \left(\frac{A}{B_{c}-B}\right)
$$

where $A$ is a constant. Note that this relaxation is the overall relaxation time for a macroscopic observable ( average current-density for an ensemble of noninteracting particle). We are not discussing the largest relaxation time, as that is infinite for all $B$, however small.

Thus, biassed diffusion of noninteracting particles on a percolation network provides a very simple model where a fast rise of relaxation time near a dynamical phase-transition (" the Vogel-Fulcher law" of glassy dynamics) can be seen.

We see that disorder effects both the static and dynamical properties of the system in a very significant way. The effect on non-equilibrium properties like response functions is much more pronounced. I hope that further work will lead to a better understanding of these systems. 


\section{References}

[1] See, for example, D. Stauffer, Phys. Rep. 54, (1979) 1; D. Stauffer and A. Aharony, Introduction to Percolation Theory, ( Taylor and Francis, London, 1991); G. Grimmett,Percolation Theory, ( Springer-Verlag, NY, 1999).

[2] This definition differs from the definition often used in literature, where $\theta$ is usually defined in terms of numbers of unrooted animals. As the number of unrooted clusters of $n$ sites is just $A_{n} / n$, this definition gives a value 1 larger than the one used by us.

[3] G. Parisi and N. Sourlas, Phys. rev. Lett., 46, (1981) 871.

[4] C. N. Yang and T. D. Lee, Phys. Rev 87, (1952) 404; T. D. Lee and C. N. Yang, Phys. Rev. 87, (1952) 410.

[5] A. R. Day and T. C. Lubensky, J. Phys. A 15, (1982) L285; J. L. Cardy, J. Phys. A 15 (1982) 593; N. Breur and H. K. Janssen, Z. Phys. B 48 (1982) 347.

[6] D. Dhar, Phys. Rev. Lett.,51 (1983) 853.

[7] H. Kunz and B. Souillard, Phys. Rev. Lett. 40,(1978) 133.

[8] T. C. Lubensky and A. J. McKane, J. Phys. A 14 ( 1981) L157.

[9] R. B. Griffiths, Phys. Rev. Lett., 23 (1969) 17.

[10] Stochastic evolution in Ising models, in Stochastic Processes: Formalism and Applications, Eds. G. S. Agarwal and S. Dattagupta, Lecture Notes in Physics 184 (Springer, Berlin, 1983), p.300.

[11] D. Dhar, M. Randeria and J. P. Sethna, Europhys. Lett., bf 5 (1988) 485.

[12] A. J. Bray, Phys. Rev. Lett. 60, (1988) 720.

[13] H. Takano and S. Miyashita, J. Phys. Soc. Japan, 64, (1995) 3688.

[14] P.G. de Gennes, La Recherche bf 7, 916 (1976); C. D. Mitescu, H. Ottavi and J. Roussenq, AIP Conf. Proc. 40, 377 (1978); S. Havlin and D. ben Avraham, Adv. Phys. 36, 395 (1987).

[15] H. Bottger and V. V. Bryksin, Phys. Stat. Solid. B113, 9 (1982).

[16] M. Barma and D. Dhar, J. Phys. C 16, 1451 (1982).

[17] D. Dhar and D. Stauffer, Int. J. Mod. Phys. C 9, 349 (1998). 


\section{Captions to figures}

Fig. 1: The phase digram of a diluted magnet. The ferromagentic, paramagnetic and Griffiths phases are denoted by PM, F, and G respectively.

Fig. 2: Schematic representation of the percolation cluster with density above the critical threshold. The heavy lines denote the dead-end branches.

Fig. 3: The average velocity $v$ as a function of the biassing field $B$. 